\title{
Editorial
}

\section{Polymer Composites for Passive Control System of Buildings}

\author{
Sumin Kim, ${ }^{1}$ Jae D. Chang, ${ }^{2}$ Geun Y. Yun, ${ }^{3}$ and Sughwan Kim ${ }^{4}$ \\ ${ }^{1}$ Soongsil University, Seoul, Republic of Korea \\ ${ }^{2}$ University of Kansas, Lawrence, KS, USA \\ ${ }^{3}$ Kyung Hee University, Yongin, Republic of Korea \\ ${ }^{4}$ Technical University of Munich, Munich, Germany \\ Correspondence should be addressed to Sumin Kim; skim@ssu.ac.kr
}

Received 9 October 2017; Accepted 11 October 2017; Published 1 November 2017

Copyright (C) 2017 Sumin Kim et al. This is an open access article distributed under the Creative Commons Attribution License, which permits unrestricted use, distribution, and reproduction in any medium, provided the original work is properly cited.

Currently, many researches of polymer and polymer-based materials have been showing a lot of valuable applications in industry fields such as the building sector. Also buildings are the major energy consumers and account for as much as $45 \%$ of global energy consumption. So the passive control system in the building environment with a high energy saving potential has become increasingly important. Recently, the use of thermal energy storage with phase change material (PCM) is considered to be one of the most important advanced technologies, and a lot of attention has been paid to utilization of the essential techniques for thermal applications, ranging from heating to cooling in buildings. The thermal energy storage system in the building can smooth temperature fluctuations, and such storage can be implemented by sensible heat, or latent heat utilization. Latent heat storage enables the passive control of the temperature in buildings. This special issue encompasses research papers addressing recent advances on composites for passive control system of buildings.

Articles presented in this special issue are related to composites and materials for passive control system of buildings, thermal energy storage system in buildings, phase change materials (PCM) combination with building materials, composites based on renewable and sustainable materials, advanced architectural renewable and sustainable technology, preparation of advanced composites and materials for buildings, and advanced materials for energy-efficient buildings. D. Lee et al. propose wood plastic composites with UV stabilizer and nano- $\mathrm{CaCO}_{3}$ and talc. M. S. Kim and Y. H. Lee investigate the shear performance of concrete plates with fiber-reinforced polymer. S. Baek and J. C. Park propose a underfloor heating system with PCM for heating energy savings. B.-E. Choi et al. numerically evaluate passive energy conservation measures in buildings. S.-H. Kim et al. develop the passive redirect lighting systems for the improvement of the passive control of buildings. Finally, J.H. Song et al. empirically validate heat transfer performance of graphite/PCM concrete materials for thermally activated building system. We hope this special issue becomes a platform for a wide range of professions to discuss recent advances in polymer composites for passive control systems of buildings.

Sumin Kim Jae D. Chang Geun Y. Yun Sughwan Kim 

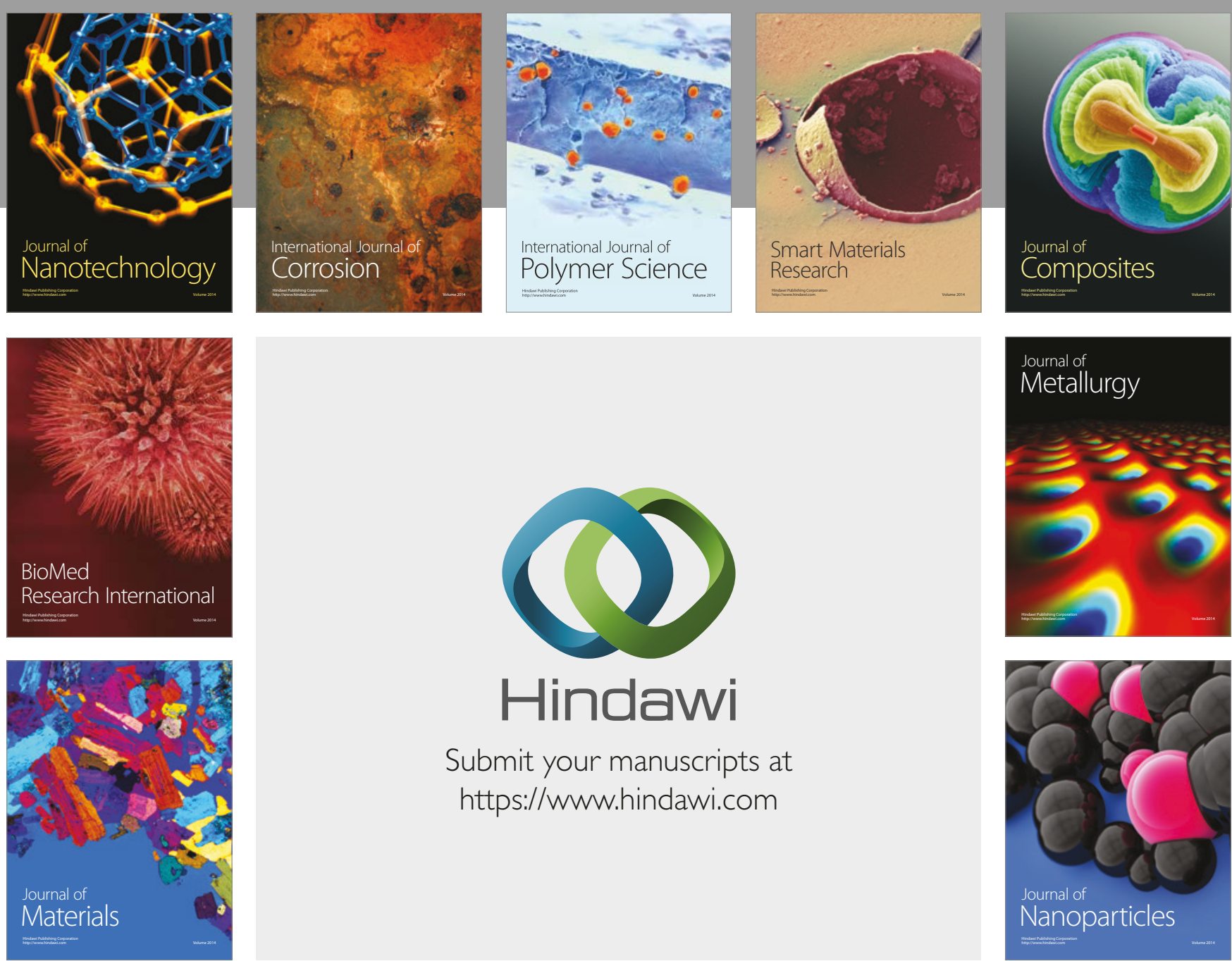

\section{Hindawi}

Submit your manuscripts at

https://www.hindawi.com
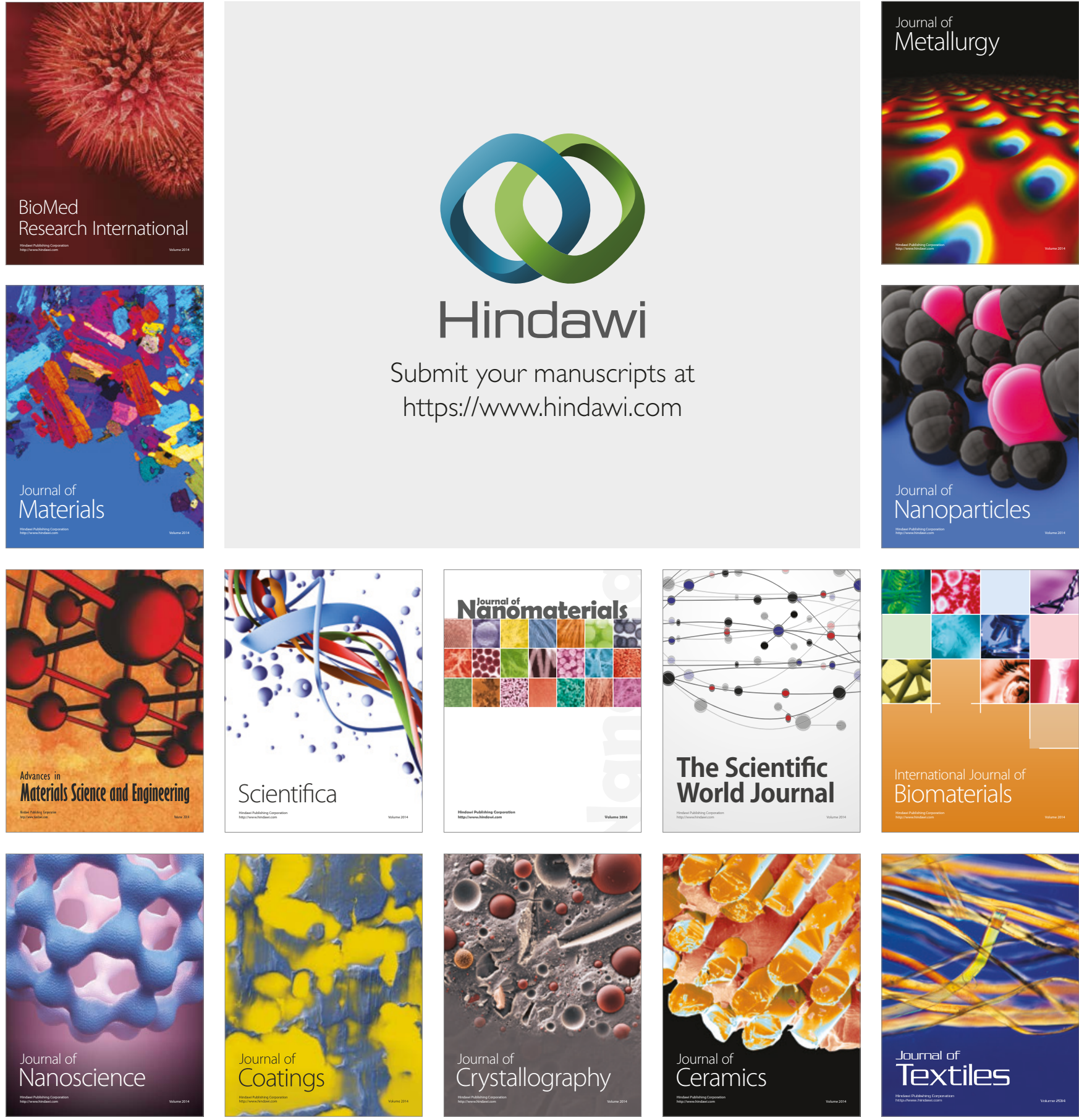

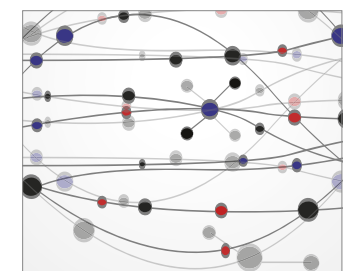

The Scientific World Journal
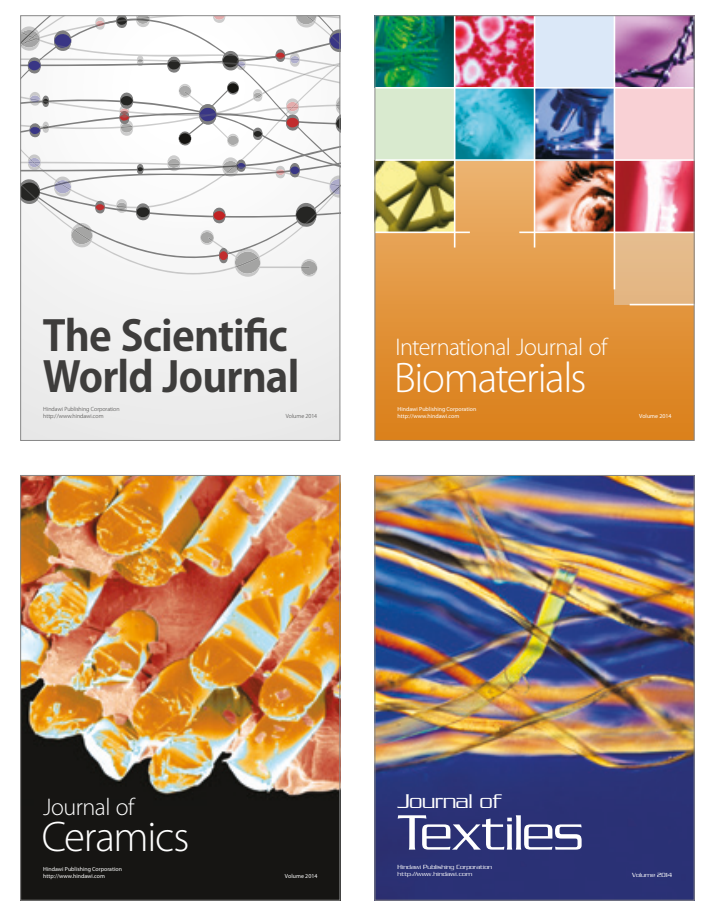